\title{
Heidelberg Laureate Forum
}

It is fall in Heidelberg and the leaves on the trees are already turning. This is the fifth year of the Heidelberg Laureate Forum (http://www.heidelberg-laureate-forum.org/)

and it continues to be a highlight of the year for me and for about 250 others who participate. This year, computer science was heavily represented. There were fewer mathematicians, but they made up for smaller numbers by their extraordinary qualifications. A new cohort of laureates was added this year: recipients of the ACM Prize for Computing. ${ }^{\text {a }}$

Quantum computing and machine learning were major foci of attention and the Hot Topic sessions drew on speakers in the research and private sectors beyond the normal cohort of laureates. I took several lessons away from the quantum computing discussions: serious progress is being made in multi-qubit hardware development; the somewhat inaptly named "Quantum Supremacy" challenge ${ }^{\mathrm{b}}$ is being met by more than one organization; and programming languages for quantum computing are being developed.

The other hot topic was machine learning and artificial intelligence and impressive results were outlined for image recognition, speech understanding, machine translation, and self-supervised learning. Multilayer neural networks are producing interesting results such as the ability to turn an ordinary photograph into one that inherits the style of various wellknown artists or schools of art. The ques-

a Formerly known as the ACM-Infosys Foundation Award in the Computing Sciences.

b That is, quantum computers in the $49-50+\mathrm{qu}-$ bit range that can theoretically perform functions beyond the capacity of current or projected conventional classical computing systems.

c https://en.wikipedia.org/wiki/Quantum_ programming\#Quantum_computing_language

tion of transparency of machine learning in which it is made clear where the deep learning takes place and what has been learned remains unanswered, as does an easy way to explain how the system has reached its conclusions. Mathematical and computational models of biological processes found their way onto the agenda, reinforcing the notion that we might someday understand better how these processes work by modeling them and predicting some of their behaviors.

Visits to local research and business centers were arranged, such as the European Microbiology Laboratory (EMBL), European Media Laboratory, German Cancer Research Center, Heidelberg Institute for Theoretical Studies, the Max Planck Institute for Astronomy, NEC, the Mathematics Center of the University of Heidelberg, and the Interdisciplinary Center for Scientific Computing and to SAP headquarters. I spent at least two hours with a researcher at EMBL discussing in depth intraand inter-cellular communication.

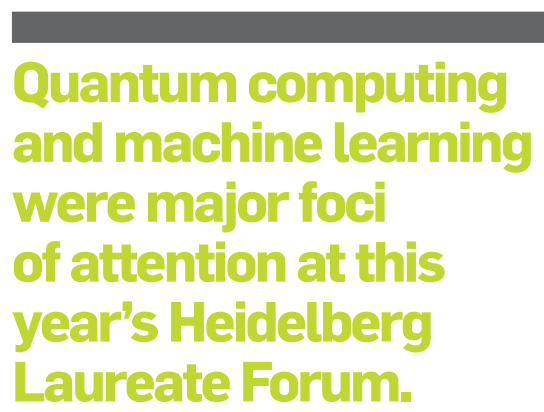

Once again, a boat trip on the Neckar River afforded several hours of free discussion among laureates and students amidst the beautiful fall colors in the trees along the journey. A Bavarian night and a visit to the amazing Speyer Museum $^{\mathrm{d}}$ also provided ample interactive opportunities in addition to which the Forum added office hours, student working groups, and discussion groups to stimulate student/laureate interactions. A final visit to the $17^{\text {th }}$ century castle overlooking Heidelberge rounded out the week.

By chance (or perhaps by careful planning), the celebration of the linking of Heidelberg to Palo Alto, CA, as sister cities was hosted in the Town Hall of Heidelberg. A number of HLF participants live or have lived in the Palo Alto area and represented the city along with its mayor and other dignitaries. Of course, the Mayor of Heidelberg and the City Council were in attendance. The stained glass walls of the ceremonial room of the Town Hall had dates in the 1300ssomething that is fairly mind-boggling for Americans whose sense of national history tends to start in the late 1700s!

This event continues to represent a remarkable gathering of brilliant and energetic minds and ACM and the other participating organizations owe a great debt to the Klaus Tschira Foundation and the Heidelberg Laureate Foundation for their adept and efficient support for this signature meeting. We remember gratefully Klaus Tschira, a founder of the German SAP company, who passed away far too soon in 2015.

d https://speyer.technik-museum.de/en/

e https://en.wikipedia.org/wiki/Heidelberg_Castle

Vinton G. Cerf is vice president and Chief Internet Evangelist at Google. He served as ACM president from 2012-2014.

Copyright held by author. 